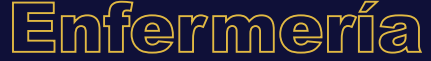

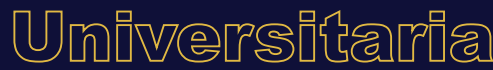

\section{Proceso de enfermería, estudio de familia de adolescente embarazada en atención primaria de salud}

\section{Nursing process, primary health care study on the family of a pregnant teenager}

\section{Processo de enfermagem, estudo de família de adolescente grávida em atenção primária de saúde}

\author{
D. Parra-Giordano ${ }^{a 1}{ }^{*}$ P. Otárola-Banda ${ }^{\mathrm{b} 2}$, V. Otay-Ríos ${ }^{\mathrm{c} 2}$ \\ ORCID: \\ ${ }^{a}$ https://orcid.org/0ooo-0oo2-9439-2679 \\ ${ }^{\mathrm{b}} \mathrm{https}$ ///orcid.org/000o-0003-3484-3156 \\ c https://orcid.org/0000-0002-1367-0914 \\ ${ }^{1}$ Universidad de Chile, Facultad de Medicina, Departamento de Enfermería, Santiago, Chile \\ ${ }^{2}$ Universidad de Chile, Facultad de Medicina, Escuela de Enfermería, Santiago, Chile \\ Recibido: 6 agosto 2019 / Aceptado: 10 diciembre 2019
}

\section{RESUMEN}

Introducción: En Chile persiste el embarazo adolescente, sin embargo, no se sistematiza la evidencia de las intervenciones realizadas, ni cómo, desde la Atención Primaria el personal de enfermería desempeña un rol fundamental en la atención de las familias para mejorar la salud de todos sus miembros.

Objetivo: Elaborar un estudio de familia de acuerdo con las directrices del proceso enfermero enmarcado en el modelo de atención integral de salud familiar y comunitaria.

Método: Desarrollo de estudio de familia con enfoque en el proceso de enfermería. Caso índice embarazada adolescente que presenta riesgo tras la aplicación de la pauta de Riesgo Psicosocial-Evaluación Psicosocial Abreviada en el control de ingreso de embarazo en el marco del programa Chile Crece Contigo.

Desarrollo: Aplicación de instrumentos de valoración familiar mediante visita domiciliaria integral, realización de proceso enfermero e intervención mediante programa educativo. Registro en ficha electrónica, elaboración de informe para seguimiento del caso por parte del equipo; evaluación de proceso, estructura y resultado para posteriormente evaluar los objetivos a corto plazo planteados en el proceso enfermero.

\footnotetext{
*Autor para correspondencia. Correo electrónico: denisseparrag@gmail.com 
Conclusiones: En el contexto del modelo de atención integral de salud familiar y comunitaria, el estudio de familia se vuelve fundamental para la comprensión holística de la familia como unidad funcional de la sociedad. Mas, es el uso de la sistematización del Proceso de Enfermería, lo que permite una identificación de la problemática de la familia y su intervención para disminuir el riesgo psicosocial de una embarazada adolescente.

Palabras clave: Adolescente; embarazo; atención de enfermería; visita domiciliaria; atención primaria de salud; Chile.

\section{ABSTRACT}

Introduction: In Chile, teenage pregnancies are still occuring, but the evidence regarding the interventions, including those from family-focussed nursing in primary health care, is not systematized.

Objective: To carry out a family study following the phases of the nursing process under the family and community health integral attention model framework.

Method: Family study following the nursing process. The index case is a pregnant teenager who, as reflected by the Pauta de Riesgo Psicosocial-Evaluación de Riesgo Psicosocial instrument from the Chile Crece Contigo program, was found to be at risk.

Development: Family assessment instruments were applied during home visits. Following the nursing process, the intervention was based on an educational program. An electronic record was registered. A follow up inform on the case was elaborated. The process, structure, and primary results were assessed to support the evaluation of the short term objectives set out in the nursing process.

Conclusions: Within the context of the family and community health integral attention model, the family study becomes fundamental in order to holistically understand the family as a functional unit of society. Therefore, the systematization of the nursing process allows a better identification of the problems within the family - in this case a teenage pregnancy, and thus the development of better approaches to reduce the related psychosocial risks.

Keywords: Adolescent; pregnancy; nursing care; house call; primary health care; Chile.

\section{RESUMO}

Introdução: No Chile persiste a gravidez adolescente, porém, não se sistematiza a evidência das intervenções realizadas, nem o como, desde a Atenção Primária o pessoal de enfermagem desempenha um papel fundamental na atenção das famílias para melhorar a saúde de todos seus membros.

Objetivo: Elaborar um estudo de família conforme às diretrizes do processo enfermeiro enquadrado no modelo de atenção integral de saúde familiar e comunitária.

Método: Desenvolvimento de estudo de família com enfoque no processo de enfermagem. O caso de índice gravidez adolescente que apresenta risco depois da aplicação da Pauta de Riesgo Psicosocial-Evaluación Psicosocial Abreviada no controle de ingresso de gravidez no marco do programa Chile Crece Contigo.

Desenvolvimento: Aplicação de instrumentos de valoração familiar mediante visita domiciliar integral, realização de processo enfermeiro e intervenção mediante programa educativo. Registro em ficha eletrônica, elaboração de relatório para seguimento do caso por parte da equipe; avaliação de processo, estrutura e resultado para posteriormente avaliar os objetivos a curto prazo levantados no processo de enfermagem.

Conclusões: No contexto do modelo de atenção integral de saúde familiar e comunitária, o estudo de família torna-se fundamental para a compreensão holística da família como 
unidade funcional da sociedade. Mas, é o uso da sistematização do Processo de Enfermagem, o que permite uma identificação da problemática da família e sua intervenção para diminuir o risco psicossocial de uma grávida adolescente.

Palavras chave: Adolescente; gravidez; atenção de enfermagem; visita domiciliar; atenção primária de saúde; Chile.

\section{INTRODUCCIÓN}

En Chile persiste el embarazo adolescente ${ }^{1}$, el cual presenta un riesgo mayor para la madre, tanto por el daño físico en el cuerpo de la diada, como psicosocial por las dificultades sociales que impli$\mathrm{ca}^{1-4}$, además del impacto económico y social en estas familias4. Ante esta problemática el rol de Enfermería es fundamental en la atención de las familias para mejorar la salud de todos sus miembros desde la Atención Primaria de Salud (APS); no obstante, la participación de este profesional no se ha sistematizado.

De acuerdo con la Organización Mundial de Salud (OMS) aproximadamente un millón de niñas menores de 15 años y 16 millones de jóvenes de 15 a 19 años dan a luz cada año ${ }^{5}$. En Chile el 10.7\% de los nacidos vivos son de madres adolescentes $(26165)^{6}$.

Las políticas públicas y programas referidos a la salud de la población chilena han priorizado la primera infancia mediante controles de salud en esta etapa de vida, por medio del sistema de protección del programa Chile Crece Contigo (ChCC)7. Sin embargo, a pesar de que los adolescentes presentan mayores condiciones de vulnerabilidad que otros grupos etarios, no se ha puesto mayor atención de este grupo en la planificación y orientación en red, por lo que existe una cobertura insuficiente de las políticas y programas de salud preventiva en adolescentes, con una baja oferta de servicios $^{8}$. Existen fallas en la atención preventiva para adolescentes de 10 a 14 años, y los escasos esfuerzos se han centrado en la población mayor de 15 años, al incluir en el año 2005 en las Garantías Explícitas en Salud (GES) el Examen de Salud de Medicina Preventiva (EMPA) ${ }^{9}$.

Conforme a este marco de la atención de salud, así como de las actividades del profesional de enfermería, que es parte esencial del equipo de trabajo en Atención Primaria de Salud (APS) ${ }^{10}$, es que se plantea realizar un estudio de familia, elaborado a partir de un grupo familiar que cursa una crisis no normativa debido al embarazo de una adolescente.

Enfermería es una disciplina profesional práctica que busca dar cuidado para la salud de personas, familias y comunidad ${ }^{11}$. La gestión del cuidado se desarrolla por medio del Proceso de Enfermería, herramienta propia de la profesión que, fundamentándose en modelos y teorías, reconoce a la persona, familia o grupo social en su contexto habitual e identifica sus características por medio de una valoración holística que le permite identificar sus necesidades afectadas, y crear un plan de cuidados que ayude a satisfacer dichas necesidades ${ }^{12}$. Por otro lado, el estudio de caso se presenta como un instrumento formativo y disciplinar que permite tanto la reflexión como el análisis de las distintas situaciones que se presentan durante la práctica, por tal razón es utilizado como fuente de aprendizaje y retroalimentación de las atenciones brindadas ${ }^{13}$.

Así, el presente informe se realiza con base en un estudio de familia; a la persona índice, quien entrega la información de la familia y en quien se centra este estudio, le corresponde ser atendida en un Centro de Salud Familiar (CESFAM) del Servicio de Salud Metropolitano Norte de la región Metropolitana de Santiago de Chile. El objetivo del presente documento es elaborar un estudio de caso de acuerdo con las directrices del proceso de enfermería y enmarcado en el modelo de atención integral de salud familiar y comunitaria. 


\section{METODOLOGÍA}

La familia por estudiar fue asignada por parte de la profesional encargada del Programa de la Mujer en el CESFAM. El grupo familiar requería una visita domiciliaria debido al riesgo presente tras la aplicación de la Evaluación Psicosocial Abreviada (EPsA) $)^{14}$, realizada en control de ingreso de embarazo a la persona índice ${ }^{15}$, que valora las siguientes condiciones: menor a 20 semanas de gestación, escolaridad menor a $6^{\circ}$ básico, edad menor a 17 años, conflictos con la maternidad, insuficiente apoyo social o familiar, síntomas depresivos, uso o abuso de sustancias, violencia de género, así como un apartado de observaciones para otros datos relevantes identificados ${ }^{14}$; necesarios para asegurar la continuidad de los controles de la embarazada durante el proceso de gestación de su primer hijo/a, en el marco del programa ChCC?.

Se desarrolló el estudio de familia conforme a las cinco etapas del proceso de enfermería ${ }^{12}$; en la etapa de valoración se realizó la recopilación de antecedentes a partir de la ficha electrónica, seguido de una visita domiciliaria, con el propósito de evaluar el entorno y funcionamiento familiar que incluyó la aplicación de instrumentos de valoración familiar. Luego del análisis de la información recopilada, en la etapa de diagnóstico, se plantearon dos diagnósticos de enfermería los cuales se centraron en los hallazgos más relevantes de la valoración. Durante la etapa de planificación, se propusieron intervenciones acordes a las necesidades encontradas y se planteó la forma de aplicarlas. En la etapa de ejecución, las intervenciones propuestas se desarrollaron en un segundo encuentro; además de la orientación educativa que se llevó a cabo en las dependencias del CESFAM. Al finalizar, durante la etapa de evaluación, se analizó la efectividad de las intervenciones, así como de los recursos empleados y se dejó registro en ficha electrónica, junto con la propuesta de elaborar un informe para un posterior seguimiento del caso por parte del equipo de trabajo del CESFAM.

Se contó con el consentimiento firmado de la gestante para la realización de la visita domiciliaria.

\section{DESARROLLO}

\section{Valoración familiar}

En cuanto al ambiente, la vivienda de la familia R.O. era de material liviano, con evidentes problemas estructurales. La valoración se realizó en la entrada externa de la casa (antejardín), se observó acumulación de materiales de construcción y basura en los rincones. La persona índice, M.R., no mencionó el número de habitaciones que componen la vivienda, sin embargo, indicó que, en la habitación del padre, F.R., se encuentran la cocina y el refrigerador, espacio que también es utilizado como comedor. El baño estaba ubicado fuera del domicilio y se encontraba en proceso de mejoramiento en su estructura y sistema de alcantarillado. El hogar contaba con los servicios básicos (luz, agua, alcantarillado y servicio de retiro de basura). La familia utilizaba la cocina y un bracero como medio de calefacción doméstica. La vivienda estaba ubicada próxima al CESFAM. En el entorno se observaron perros vagos, focos de basura, ausencia de áreas verdes y escasa movilización.

\section{Estructura familiar}

La estructura familiar se describe en el genograma familiar (Figura 1). El genograma utilizado en Chile es el descrito por McGoldrick (citado por Suárez-Cuba) que entrega una representación gráfica familiar que da a conocer la estructura, relaciones y/o funcionalidad de sus integrantes con un mínimo de tres generaciones ${ }^{16-18}$. Al analizar este instrumento de valoración se aprecian las distintas relaciones que la persona índice tiene con los integrantes de su familia; asimismo, una relación estrecha con su hermana mayor J.R. madre de dos menores, y V.R. hermana menor, además de la 
relación con su pareja B., quien es su principal red de apoyo. Mantiene relaciones cercanas con su padre y sobrinos. Existe un quiebre en la relación con su madre como consecuencia del consumo de alcohol y drogas por parte de la madre. Cabe destacar que la madre pernocta en el domicilio familiar.

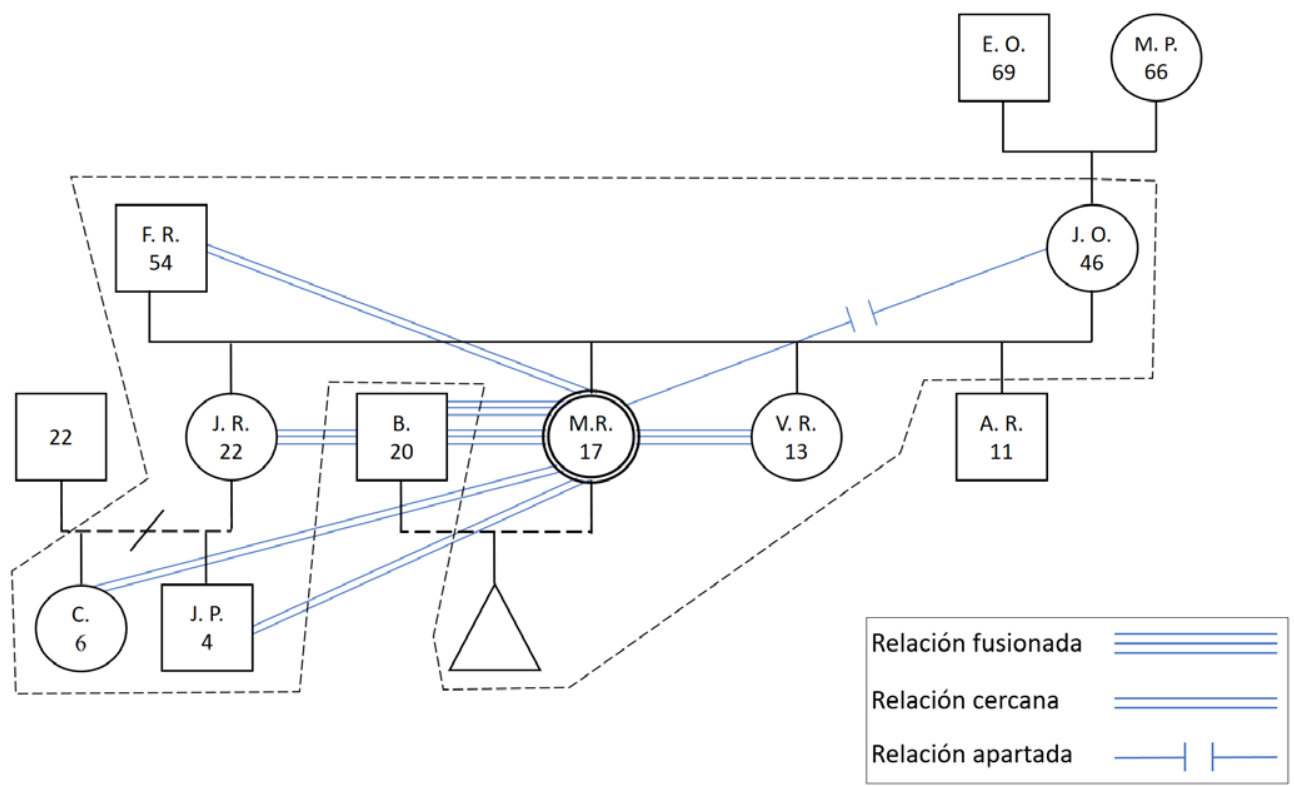

Figura 1. Genograma familiar

Se construyó también el ecomapa familiar (Figura 2), el cual permite representar de forma dinámica el sistema que rodea a la familia, refleja sus conexiones y relación con la sociedad, las cuales pueden ser útiles en momentos de crisis ${ }^{16,17,19}$. Éste evidencia la limitada red de apoyo que posee la familia. M.R. mantiene una interacción débil con el CESFAM, a pesar de su embarazo y de la cercanía del recinto con su hogar, pues se encontraba ausente a controles. La persona índice además asiste a la Dirección de Educación Municipal (DEM) con el fin de regularizar sus estudios, esto debido a que en el pasado desertó del sistema educacional. Se evidencia un desinterés en su discurso, pese a que el establecimiento le otorga las facilidades para finalizar su educación básica. Todos los menores de la familia tienen una relación con establecimientos educacionales. Respecto al ámbito laboral, F.R. y J.R. trabajan como vendedores en un mercado ambulante de frutas y verduras.

Para concluir, la persona índice M.R. dibujó el círculo familiar (Figura 3), el cual representa de forma gráfica y esquemática la percepción que tiene la persona que lo dibuja de sus relaciones con los miembros de la familia; ayuda tanto al profesional como a la persona a comprender las relaciones internas de su núcleo ${ }^{16,20}$. De acuerdo con lo anterior y según indicó la persona, ésta no otorgó un ordenamiento significativo de los nombres, pero de acuerdo con su interpretación se puede decir que M.R. ve como un apoyo importante a la madre y abuela de su pareja; menciona que no sólo se preocupan de ella, sino también de sus hermanas y sobrinos. Luego se aprecia a V.R. y sus sobrinos que representan su compañía diaria. En cuanto a su padre y pareja, los ubica en una línea secundaria, sin embargo, indica que B. es su red de apoyo más importante. Finalmente se observa a su hermana mayor J.R., de la cual menciona no sentir tanto apoyo. Es importante destacar que M.R no considera a su madre dentro del círculo; no existe relación alguna con ella. 


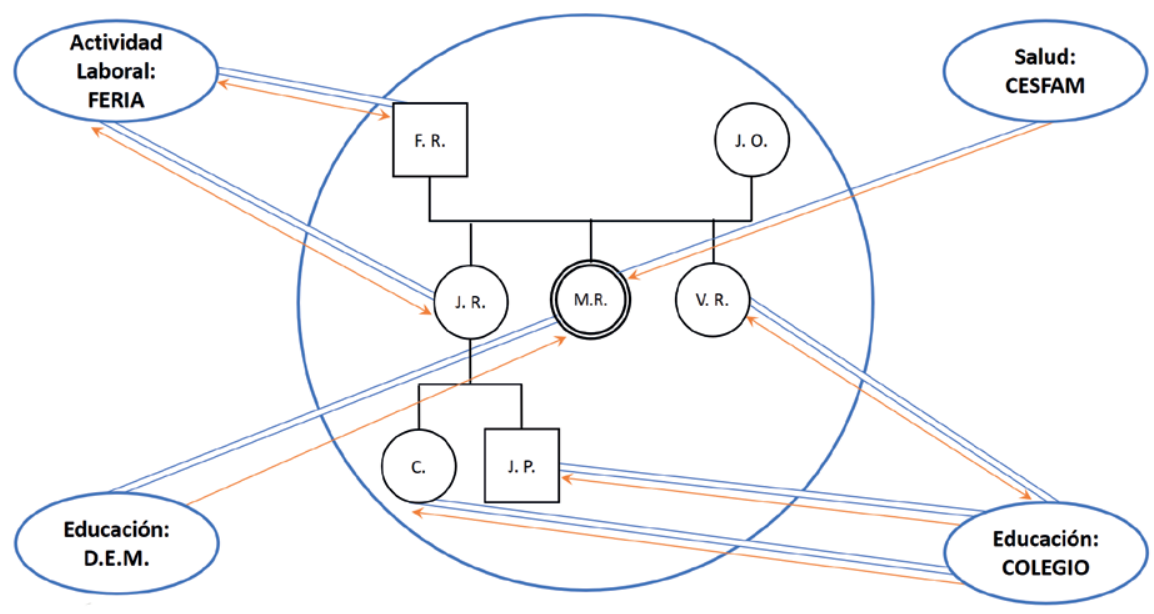

Figura 2. Ecomapa familiar

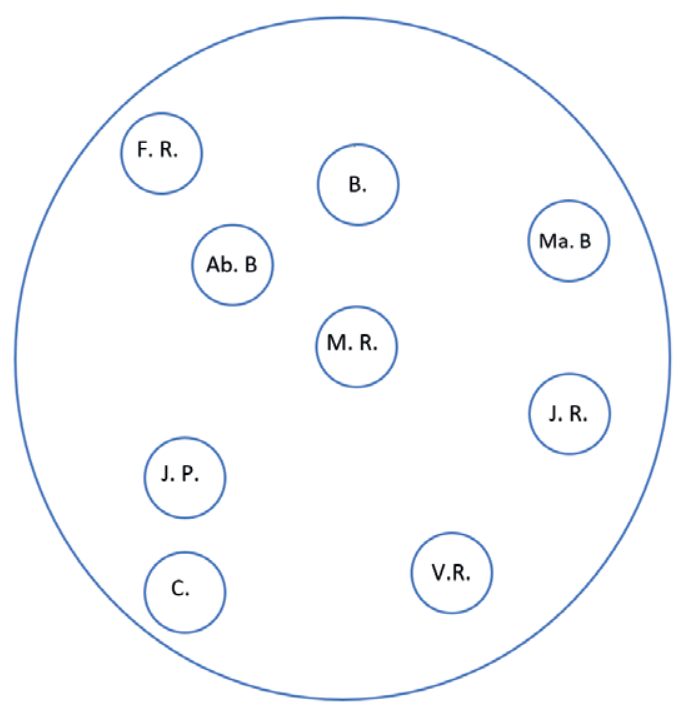

Figura 3. Círculo familiar

Para complementar la valoración de la familia se aplicó el APGAR familiar diseñado por Smilkstein y validado en Chile por Florenzano et al., el cual consiste en un cuestionario autoadministrado de cinco preguntas cerradas que reflejan la percepción y el grado de satisfacción que tiene la persona del funcionamiento familiar. El puntaje obtenido permite clasificar la percepción del caso índice de su familia como moderadamente funcional. Este instrumento permite evaluar el funcionamiento familiar en cinco grandes áreas: adaptabilidad, participación, desarrollo, afectividad y capacidad de resolución $n^{16,17,21 .}$ 


\section{Funcionalidad familiar}

Se entiende como la capacidad del núcleo de enfrentar y superar las diversas etapas que atraviesan a lo largo del tiempo. Existen funciones básicas familiares establecidas por el Ministerio de Salud de Chile ${ }^{17}$.

Función biológica. J.R., hermana mayor de la persona índice, fue madre de su primera hija durante la adolescencia. M.R. actualmente cursa la semana 14 de su embarazo. Respecto a la crianza de los hijos F.R. es un expresidiario y se encontraba privado de libertad hasta aproximadamente un año, razón por la cual no fue partícipe activo de la crianza de sus hijos menores. Por otra parte, la adicción de J.O., madre de la persona índice, propició el alejamiento del grupo familiar. Ante esta situación la abuela paterna se hizo cargo de la crianza de los menores del grupo familiar.

Función educativa-socializadora. Los menores asisten a instituciones educacionales subvencionadas total o parcialmente por el estado. M.R. suspendió su asistencia a centros educacionales en el pasado y actualmente cursa los niveles correspondientes a séptimo y octavo de la educación básica del sistema educacional chileno. No existen redes de apoyo vecinales o familiares; la única red de apoyo que M.R. describe es su pareja y su familia.

Función económica. No es posible asegurar un ingreso económico estable por parte de los miembros de la familia; el único ingreso estable lo aporta B., pareja de M.R. y padre de su hijo en gestación. Las necesidades básicas como alimentación, vivienda, vestimenta, así como servicios básicos y sanitarios se cumplen total o parcialmente; de igual forma la familia es capaz de sustentar a sus miembros no productivos y lograr la distribución de labores domésticas.

Función solidaria. M.R. expresa que su principal red de apoyo es su pareja, además de sus sobrinos y hermana menor de quienes ella es la principal cuidadora. La familia no realiza actividades en conjunto. Las labores domésticas son asumidas por M.R. Aun cuando J.O. reside en el hogar familiar, no realiza ningún tipo de aporte al grupo familiar.

Función protectora (Psicológica). M.R. es la principal cuidadora de su hermana menor y sobrinos, con los cuales tiene una relación de cercanía. Menciona que no siente sus necesidades de afecto satisfechas por su familia.

Por otra parte, según el modelo de Duvall ${ }^{16,22}$, la familia se encuentra en la etapa del ciclo vital de familia como plataforma de lanzamiento, que comprende el periodo desde los veinte años del primogénito/a hasta que el último hijo abandona el hogar familiar; de acuerdo con esto las tareas de la etapa las enfrentan como se describe a continuación:

Tolerancia a la partida de los hijos. Padre y madre de esta familia deben estar dispuestos y aceptar el hecho de que, en algún momento, sus hijas quieran partir del hogar y empezar una nueva vida. Es necesario el diálogo previo a cambios para que se puedan enfrentar de la mejor manera. En el caso particular de la familia, la primogénita vive en el hogar familiar junto a sus dos hijos y aún no proyecta su salida del hogar. Por otro lado, la persona índice menciona que su pareja se irá a vivir con ella a la casa una vez que nazca su hijo.

Independencia de los hijos, elección de pareja y vocacional. J.R. tiene cierto grado de independencia, ya que tiene a sus hijos además de ser la mayor de los hermanos. A pesar de esto vive con sus padres y utiliza todos los ingresos económicos que logra generar en el sustento del hogar familiar, sin recibir ayuda económica externa al núcleo familiar. La persona índice tiene una relación de larga data con su pareja y juntos tendrán un bebé; sin embargo, ésta aún sigue bajo el cuidado y las restricciones que su padre le indica. Respecto a la elección vocacional, la familia debe estar abierta 
a la posibilidad de que los hijos quieran estudiar de forma profesional. En el caso de dicha familia, su deber es impulsar y motivar a sus hijas a continuar hasta finalizar su educación básica y media, según el modelo educacional chileno.

Cambios en los limites intergeneracionales, apertura a los límites familiares. Aceptación de los nuevos miembros de la familia y refuerzo de los lazos entre ellos para que las relaciones se fortalezcan. En este caso, próximamente se integrarán dos nuevos miembros, el bebé de la persona índice y su pareja, por lo cual el resto de la familia debe aceptar esta situación para evitar problemas de convivencia. Es importante establecer roles para que exista respeto en el desarrollo de estos.

\section{Crisis}

Junto con lo anteriormente dicho, es importante recalcar que durante su ciclo las familias deben enfrentarse a una serie de cambios dados por el desarrollo del grupo familiar. Estos cambios o crisis pueden clasificarse como predecibles del desarrollo normal de una familia o normativas y, por otro lado, ser impredecibles, circunstanciales o no normativas ${ }^{23-26}$; del mismo modo, es importante destacar que las crisis no dependen de la funcionalidad o disfuncionalidad de una familia, pero sí de las herramientas para afrontarlas ${ }^{26}$.

Crisis normativas. Son esperables durante el ciclo familiar; la familia debe cumplir ciertas tareas correspondientes a la etapa que cursa dentro de este ciclo, para así promover la adaptación estructural y funcional que permita el desarrollo familiar ${ }^{23-27}$.

Adolescencia. Durante el desarrollo del ciclo familiar se espera que los hijos cursen por el período de la adolescencia, que trae consigo una serie de cambios físicos y psicológicos que pueden resultar difíciles de afrontar para la persona en desarrollo, razón por la cual la familia debe adaptarse a estos cambios y generar respuestas que permitan mantener la funcionalidad familiar. En el caso de la familia R.O., su hija menor V.R. está en las etapas iniciales de la adolescencia, además M.R. ya cursa por esta etapa del ciclo vital, requiere cuidados y un proceso de adaptación propio orientado a sus necesidades de mujer gestante. Asimismo, se enfrentará a la crisis de pareja frente al nacimiento de un hijo, cambiando los roles y la distribución de los tiempos ${ }^{26}$, lo cual es importante a considerar siendo la pareja el principal apoyo de la gestante.

Crisis no normativas. Son de aparición abrupta y requieren una respuesta rápida del grupo familiar para su manejo. En ocasiones pueden ser solucionadas de forma favorable, pero en otras la familia debe generar cambios en su estructura, roles y comunicación con el fin de resolver en conjunto las distintas situaciones que se presenten ${ }^{23-25}$.

Embarazo adolescente. M.R. cursa la décimo cuarta semana de gestación a la edad de 17 años, esta situación requiere una adecuación del grupo familiar adaptando el ambiente de la vivienda para satisfacer las necesidades de la madre y el hijo en gestación, brindándoles un ambiente seguro.

Finalmente, se identifican factores propios de la familia que influyen en el funcionamiento familiar, en forma negativa:

Nivel de escolaridad. M.R., la persona índice, de 17 años aún no concluye la enseñanza básica y en el pasado suspendió su asistencia a centros educacionales. Actualmente asiste a la DEM para cursar niveles análogos a séptimo y octavo de educación básica, pero con un hijo en gestación existe la posibilidad cierta de que deserte del sistema educacional, situación que perpetuará el círculo de la pobreza, y por ende seguir manteniendo las características sociodemográficas que caracterizan a este grupo $^{24}$. La hermana menor de M.R. cursa niveles inferiores a los que le corresponden según su edad. 
Embarazo adolescente. Es un factor de riesgo debido a su categoría de crisis no normativa dentro de la familia.

Por otra parte, como factores familiares que influyen positivamente en el funcionamiento familiar tenemos:

Red de apoyo familiar presente. Se considera como factor protector ya que se relata por parte de los integrantes de la familia que frente a problemas se apoyan; al conocer el estado de embarazo de las hijas siempre se les ayudó y estuvo con ellas.

Apoyo por parte de la familia de B. Es un factor protector ya que siempre se preocupan por la salud de M.R. y de su familia. M.R. manifiesta que en ocasiones cuando no tienen qué comer, la abuela o madre de B. los invitan a su casa. También B. los ayuda económicamente y brinda a su pareja contención emocional.

Educación, DEM. Es un apoyo para la familia, ya que le da las herramientas a M.R. para que continúe con sus estudios, le da protección necesaria y facilidades para que pueda continuar educándose pese a su embarazo. También se le asigna a M.R. un tutor, quien la ayuda si necesita algo y esto también beneficia a la familia.

\section{Diagnósticos de enfermería}

Como describe $T^{2} l l e z^{28}$, los diagnósticos reales se forman a partir de tres componentes: problema, factores relacionados o etiología y, signos/sintomas o características definitorias. De igual forma, menciona que los diagnósticos NANDA emplean el formato PES (P: problema, E: etiología, S: signos y síntomas), se considera el problema como la etiqueta diagnóstica NANDA, la etiología el factor relacionado, y los signos/síntomas las características definitorias.

Se jerarquiza un diagnóstico individual y un diagnóstico familiar reales para intervenir.

De acuerdo con esto se utiliza como base para el diagnóstico individual la etiqueta NANDA 00058 (Riesgo de deterioro de la vinculación) para la confección del diagnóstico; sin embargo, se adapta al formato PES, el cual es el utilizado en la Escuela de Enfermería de la Universidad de Chile. Por otro lado, el diagnóstico familiar expuesto sigue el formato CIPE basándose en la valoración realizada e instrumentos aplicados (Tabla 1).

Cabe mencionar que dentro de la ejecución de este estudio sólo se desarrolló el diagnóstico PES.

\section{Planificación de enfermería}

La planificación de enfermería queda evidenciada en la Tabla 1, la cual refleja el trabajo en continuidad que busca otorgar la atención primaria de acuerdo con las competencias de los estudiantes de enfermería en formación. Se establece objetivo a corto plazo el que es evaluado durante la intervención realizada en el CESFAM, además de objetivos a mediano y largo plazo que se alcanzarán con el tiempo.

\section{Ejecución de enfermería}

Las actividades de la intervención se llevan a cabo en el CESFAM junto a la persona índice M.R., tal y como se acordó durante la visita domiciliaria. Se destaca que a la orientación educativa realizada también asiste B., pareja de M.R., quien tuvo una recepción buena durante el desarrollo de la actividad, la cual contemplaba el apego que tiene que existir entre madre y bebé desde antes de que éste nazca. Durante el desarrollo de la orientación M.R. se muestra reacia, por lo que es reconvenida e 
invitada a participar en la educación. Se logran realizar todas las intervenciones que se tenían contempladas, estas pueden constituir una nueva herramienta para el futuro de los padres, la evaluación se presenta en el siguiente paso. Cabe destacar que las intervenciones para lograr los objetivos a mediano y largo plazo mencionadas en la Tabla 1, serán realizadas por el CESFAM según las coordinaciones propias que se hagan con la persona índice durante sus futuros controles.

\section{Tabla 1: Diagnósticos y planificación del proceso de enfermería}

Diagnóstico individual:
Riesgo de deterioro de la
vinculación materno-fetal
relacionado con déficit de
conocimiento, manifestado
por verbalización sobre
el desconocimiento de
su estado de embarazo y
desarrollo fetal.

desarrollo fetal.
Objetivo general:

- M.R. logrará establecer una vinculación materno-fetal segura con su hijo desde los primeros meses de embarazo.

Objetivos especificos:

Corto plazo:

- Conocer qué es el apego y la importancia de este en el desarrollo del menor.

Mediano plazo:

- Implementar acciones que favorecen la relación de apego.

Largo plazo:

- Realizar actividades de forma consistente en el tiempo con el fin de fortalecer la vinculación de la diada.

Intervenciones a corto plazo:

- Identificar temores, dudas o inseguridades que la persona refiera respecto a la maternidad y embarazo.

- Realizar educación en torno al apego y su importancia en el desarrollo.

- Entregar díptico educativo Apego Seguro.

- Entregar CD de estimulación prenatal como parte del programa de protección a la infancia ChCC.

- Aplicar pauta de apego seguro a los 4 y 12 meses como parte de los controles de salud realizados en la red de atención primaria en salud.

- Resolver dudas que se presenten durante las intervenciones.

Intervenciones a mediano y largo plazo:

- Educar sobre técnicas de apego y cómo llevarlas a cabo.

- Realizar un seguimiento y evaluación del apego durante los controles posteriores.

- Aplicar escala de valoración Massie-Campbell.

Diagnóstico familiar: Objetivo general:

CIPE Familia vulnerable relacionado con comunicación familiar ineficaz y dificultad para reconocer redes de apoyo externas, manifestado por Ecomapa en el que se aprecia relaciones débiles con CESFAM y APGAR, lo que evidencia una familia moderadamente funcional.

Objetivos especificos:

Corto plazo:

- Conocer el concepto y características de la comunicación y vinculación familiar con el CESFAM.

Mediano plazo:

- Tomar conciencia sobre la importancia de la comunicación familiar y vinculación familiar con el CESFAM.

Largo plazo:

- Implementar acciones de forma consistente en el tiempo, con el fin de

Intervenciones a mediano y largo plazo:

- Realizar consejería en temas de interés familiar.

- Aplicación de APGAR a integrantes de la familia para reevaluación.

- Evaluar asistencia a controles programados según necesidad. perpetuar la vinculación con el CESFAM y favorecer la comunicación familiar.

Intervenciones a corto plazo:

- Realizar visita domiciliaria para aplicar APGAR a los otros integrantes de la familia.

- Entregar material informativo con las redes de apoyo cercanas a las cuales pueden acudir.

- Derivación a profesional de salud mental según necesidad familiar. 


\section{Evaluación de enfermería}

Para la evaluación del diagnóstico (Tabla 2), se contempla la estructura de la intervención a realizar que abarca los recursos humanos, el tiempo y el material necesario para la misma. En el proceso se evaluaron las distintas actividades que se llevaron a cabo durante la intervención. Finalmente, para la evaluación de resultados se generaron indicadores, destaca que sólo fueron evaluados los de corto plazo.

\section{Limitaciones}

Se destaca el hecho de sólo haber realizado la valoración externa de la vivienda y tener acceso a únicamente dos de los integrantes de la familia.

Tabla 2. Evaluación del proceso de enfermería

Riesgo de deterioro de la vinculación materno-fetal - Se contó con recurso humano de los estudiantes y relacionado con déficit de conocimiento, manifestado por verbalización sobre el docente. desconocimiento de su estado de embarazo y desarrollo fetal.

- Se contó con recurso humano del educando.

Recurso tiempo:

- Educando se presenta a la hora acordada.

- Se contó con los materiales destinados a la intervención (Díptico, guías y CD de estimulación prenatal).

Indicadores de proceso:

- Se planifica el desarrollo de la educación.

- Se explica la importancia del tema a desarrollar.

- Se logra realizar entrega del material.

Indicadores de resultado (Objetivo a corto plazo):

- Verbaliza qué es el apego y la importancia de este en el desarrollo del menor*.

Indicadores de resultado (Objetivos a mediano y largo plazo):

- Realizar acciones que favorecen la relación de apego.

- Realización mantenida de las actividades que fortalecen el apego.

Diagnóstico familiar:

Familia vulnerable relacionado con dificultad para reconocer redes de apoyo externas y comunicación familiar ineficaz, manifestado por Ecomapa en el que se aprecia relaciones débiles con CESFAM y APGAR, lo que evidencia una familia moderadamente funcional.
Indicadores de estructura:

- Se contó con recurso humano profesional.

- Se contó con recurso humano del educando.

- Se contó con los materiales destinados a la intervención.

Indicadores de proceso:

- Se planifica el desarrollo de la intervención/educación.

- Se explica la importancia del tema a desarrollar.

- Se logra realizar entrega del material.

Indicadores de resultado (Objetivo a corto plazo):

- Integrantes de la familia contestan APGAR familiar.

- Integrantes de la familia asisten a controles de salud acordados.

- Verbalizan el concepto y características de la comunicación y vinculación familiares con el CESFAM.

Indicadores de resultado (Objetivos a mediano y largo plazo):

- Realización de consejería familiar.

- Resultados del APGAR suban un punto en las categorías relacionadas con la comunicación familiar.

* Cumplimiento de objetivo a corto plazo conforme a indicador de resultado alcanzado durante la intervención. 


\section{CONCLUSIONES}

En el marco del modelo de atención de salud familiar, las herramientas de valoración como son los estudios de familia, se vuelven fundamentales a la hora de lograr una comprensión holística de esta como unidad funcional de la sociedad. Es clave la participación y compromiso de un equipo de salud interdisciplinario que sea capaz de brindar atenciones y cuidados psicosociales, que centre su quehacer en las necesidades de la familia, empodere a sus miembros y promueva la funcionalidad familiar.

En el presente estudio de caso se consideran los riesgos individuales de la persona índice en el contexto de su embarazo pues es una adolescente, con todas las repercusiones para su desarrollo futuro y el del bebé; se identificaron además factores familiares y del entorno que puedan afectar el desarrollo de su embarazo.

Detectar factores de riesgo y protectores de forma temprana permite tener una herramienta que a futuro logrará la continuidad del cuidado, enfocándose en todos los aspectos de salud que el CESFAM pueda ayudar a resolver y contribuya de igual forma al fortalecimiento de la red de apoyo de todo el grupo familiar.

Junto con lo anterior, se destaca la importancia de la visita domiciliaria como integración al quehacer de los profesionales de la salud que permite evaluar a la persona y familia en su contexto habitual, donde se desenvuelven a diario. Si bien es cierto que en esta oportunidad no se pudo conocer el interior de la vivienda, el sólo hecho de haber estado en ella y poder observar el entorno físico más próximo es un complemento a la valoración.

Se considera fundamental para el logro de los objetivos establecidos el desarrollo de un proceso enfermero sólido, ya que en el curso de este estudio se pudieron seguir todas las etapas que dieron coherencia y continuidad al cuidado para ser programado y evaluado. Además de la conformación de un equipo sanitario interdisciplinario y comprometido con el modelo de atención de salud familiar, que brinde coherencia a las intervenciones y cuidados entregados.

\section{RESPONSABILIDADES ÉTICAS}

Protección de personas y animales. En este estudio no se realizaron experimentos con seres humanos ni animales.

Derecho a la privacidad y consentimiento informado. Conforme a lo requerido por el CESFAM se contó con consentimiento verbal para la realización del estudio de familia. En cuanto a la visita domiciliaria, se destaca que al momento del ingreso al Programa ChCC se solicita el consentimiento firmado a la gestante para la realización de visita domiciliaria en caso de ser necesario, por lo que la persona índice ya contaba con conocimiento previo de una eventual visita domiciliaria y su consentimiento al respecto.

Confidencialidad. Tanto el resguardo de la identidad de los participantes como la información entregada por ellos, ha sido manejada de acuerdo con las normas del CESFAM, se da fe que en este estudio no se presentan datos que permitan identificar a los involucrados.

Conflicto de intereses. Los autores declaran no tener conflictos de intereses.

Financiamiento. Ninguno.

\section{REFERENCIAS}

1. Manrique R, Rivero A, Ortunio M, Rivas M, Cardozo R, Guevara H. Parto pretérmino en adolescentes. Rev Obstet Ginecol Venez. 2008; 68(3): 141-3. http://bit.ly/38jbvdV 
2. Wolff C, Valenzuela P, Esteffan K,Zapata D. Depresión posparto en el embarazo adolescente: análisis del problema y sus consecuencias. Rev. Chil. Obstet. Ginecol. 2009; 74(3): 151-8.

http://dx.doi.org/10.4067/So717-75262009000300004

3. Ávalos DS, Recalde F, Cristaldo C, Puma AC, López P, Carbonell LA. Estrategia de unidades de salud familiar: su impacto en la tasa de embarazo en adolescentes en Paraguay. Rev Panam Salud Pública 2018; 42: e59. https://doi.org/10.26633/RPSP.2018.59

4. Azevedo JP, Favara M, Haddock SE, Lopez-Calva LF, Müller M, Perova E. Teenage pregnancy and opportunities in Latin America and the Caribbean: on teenage fertility decisions, poverty and economic achievement. Washington: World Bank; 2012. https://bit.ly/2NOUhoo

5. World Health Organization. Adolescent pregnancy. Geneva: WHO; 2018. https://bit.ly/2NO1H3E

6. Ministerio de Salud de Chile, Departamento de Estadísticas e Información en Salud (DEIS). Estadísticas de natalidad; nacidos vivos inscritos según edad de la madre, según región de residencia habitual de la madre y sexo del nacido. Santiago, Chile: Minsal; 2015. https://bit.ly/2Kr6Ah8

7. Ochoa G, Maillard C, Solar X. Primera infancia y políticas públicas, una aproximación al caso del Sistema Integral de Protección a la Infancia Chile Crece Contigo. Santiago, Chile: Germina. 2010. https://bit.ly/2NQ4gSJ

8. Ministerio de Salud de Chile, Subsecretaria de Redes Asistenciales. Orientaciones para la Planificación y Programación en Red 2019. Santiago, Chile: MINSAL; 2018. https://bit.ly/2Okwbt6

9. Ministerio de Salud de Chile. Guía Clínica Examen de Medicina Preventiva. Santiago, Chile: MINSAL; 2013. https://bit.ly/2qheS4f

10. Parra D. Revolución científica de la salud familiar en Chile. Rev Enf. 2016; 31: 1-16. http://dx.doi.org/10.15517/REVENF.VoI31.23159

11. Urra E. Avances de la ciencia de enfermería y su relación con la disciplina. Cienc enferm. 2009; 15(2): 9-18. http://dx.doi.org/10.4067/So717-95532009000200002

12. Reina NC. El proceso de enfermería: instrumento para el cuidado. Umbral científico. 2010; 17(1): 18-23. https://bit.ly/2NUyYuh

13. Orkaizagirre-Gómara A, Amezcua M, Huércanos-Esparza I, Arroyo-Rodríguez A. El Estudio de casos, un instrumento de aprendizaje en la Relación de Cuidado. Index Enferm. 2014; 23(4): 244-9. http://dx.doi.org/10.4321/S1132-12962014000300011

14. Ministerio de Salud de Chile. Chile Crece Contigo. Pauta de Riesgo Psicosocial Evaluación Psicosocial Abreviada (EPsA). Santiago, Chile: Minsal; 2010. https://bit.ly/2QtYUP7

15. Ministerio de Salud de Chile. Chile Crece Contigo. Manual de Atención Personalizada del Proceso reproductivo. Santiago, Chile: Minsal; 2008. https://bit.ly/2QlSHEI

16. Dois-Castellón A, Ojeda-Cabrera I, Vargas-Palavicino I, Larrea-Ouintanilla M, Quiroz-Olave M, Rodríguez-Cares $\mathrm{M}$, et al. Orientaciones para la Implementación del Modelo de Atención Integral de Salud Familiar y Comunitaria. Santiago, Chile: Minsal; 2013. https://bit.ly/2NQDgml

17. Depaux R, Campodónico L, Ringeling I, Segovia I. En el Camino a Centro de Salud Familiar. Santiago, Chile: Ministerio de Salud; 2008. https://bit.ly/341NTZj

18. Suarez Cuba MÁ. El genograma: herramienta para el estudio y abordaje de la familia. Rev Médica Paz. 2010; 16(1): 53-7. https://bit.ly/2qW31bX

19. Suarez-Cuba MÁ. Aplicación del ecomapa como herramienta para identificar recursos extrafamiliares. Rev Médica Paz. 2015; 21(1): 72-4. https://bit.ly/353Dzjo 
20. Subsecretaría de Redes Asistenciales. División de Gestión de la Red Asistencial. Modelo de Atención Integral de Salud. Serie Cuadernos Modelo de Atención $\mathrm{N}^{\circ}$ 1. Santiago, Chile: Ministerio de Salud; 2005. https://bit.ly/35aKbML

21. Montero-Ossandon L. Evaluación del funcionamiento familiar I: Genograma y APGAR Familiar. En: Dois-Castellón A, Montero-Ossandón L. Manual de Atención de Familias para Profesionales de la Salud. Santiago, Chile: Ediciones UC; 2012. p. 117-35.

22. Montero Ossandon L. Teoría Evolutiva de la familia: Ciclo vital familiar. En: Dois Castellón A., Montero Ossandón L. Manual de Atención de Familias para Profesionales de la Salud. Santiago, Chile: Ediciones UC; 2012. p. 69-85.

23. McCubbin MA, McCubbin HI. Family coping with illness: The resiliency model of family stress, adjustment and adaptation. En: Danielson CB, Winstead-Fry P,Hamel-Bissell BP(Eds). Families, health \& illness: perspectives on coping and intervention. St Louis MO: Mosby; 1996. p. 21-64.

24. Valdes-Cuervo AA. Crisis normativas y no normativas del desarrollo familiar. En: Valdés A, Ochoa J. (Eds). Familia y crisis. Estrategias de afrontamiento. México: Pearson; 2010. p. 1-10.

25. Valdez-Cuervo AA, Ochoa Alcántar JM. Familia y crisis. Estrategias de afrontamiento. México: Prentice Hall; 2010.

26. Vera-Noriega JÁ, Hurtado-Abril MF. Familia y unidades domesticas: la guerra y la paz. Ra Ximhai Rev Científica Soc Cult Desarro Sosten. 2010; 6(1): 149-52. https://bit.ly/2OkCtZK

27. Jiménez-Arrieta $M$, Amarís-Macías $M$, Valle-Amaris $M$. Afrontamiento en crisis familiares: El caso del divorcio cuando se tienen hijos adolescentes. Rev Científica Salud Uninorte. 2012; 28(1): 99-112. https://bit.ly/2KrFeYb

28. Ramírez TC, Tellez-Ortíz S. Aplicación taxonómica: NANDA, NIC y NOC en los planes de cuidados de enfermería. En: Tellez-Ortíz S, García-Flores M. Modelos de cuidados en enfermería: NANDA, NIC Y NOC. México: Mc Graw Hill; 2012. p. 87-115. 